\title{
LUMINOSITY TUNING BUMPS IN THE CLIC MAIN LINAC
}

\author{
P. Eliasson, CERN (Switzerland) and ISV, Uppsala University (Sweden); \\ D. Schulte, CERN (Switzerland)
}

\section{Abstract}

Preservation of beam emittance in the CLIC main linac is a challenging task. This requires not only beam-based alignment of the beam line components but also the use of emittance tuning bumps. In this paper the potential use of luminosity tuning bumps is explored and compared to emittance tuning bumps.

\section{INTRODUCTION}

In CLIC sophisticated beam-based alignment is required in order to preserve the beam emittance during the transport through the main linac [1]. After this procedure the remaining emittance growth is still unacceptable, mainly due to wakefield kicks from the misaligned accelerating structures. Therefore, a final stage of beam-based correction is applied; the optimisation of so-called emittance tuning bumps. Each of these bumps consists of two accelerating structures which are ideally separated by $90^{\circ}$ betatron phase advance. These structures are followed by a transverse position feedback and an emittance measurement station. The bumps are optimised by moving the structures transversely thus applying a wakefield kick to the beam. This wakefield kick can cancel the integrated kicks of the misaligned structures. The beam is resteered to the original trajectory using the feedback. The emittance measurement station is used to determine which transverse position of the structures of the bump yields the smallest emittance.

In this paper we are considering another implementation of the emittance tuning bumps in which the measurement is not performed directly after the bump but at the end of the linac. This allows correction of the emittance at a more relevant position. It also implies that the operation of each bump is no longer independent of the others.

A further variation of the tuning bumps is also considered, in which the aim is to optimise the luminosity directly instead of minimising the emittance of each of the two linacs individually. While this introduces some dependence of the bumps of each linac on the ones of the other, it optimises the most relevant value directly. Studies have indicated that the luminosity can be tuned using fast and very visible signals from the beam-beam interaction [2].

Whereas in previous studies a single luminosity tuning bump has been used, in this report the use of multiple bumps is investigated.

\section{THE TUNING BUMPS}

As mentioned above each of the tuning bumps consists of two accelerating structures ideally separated by $90^{\circ}$. In CLIC, however, a convenient choice is instead a phase advance separation of $72^{\circ}$. This corresponds to the phase advance per FODO cell. By using structures positioned in a region of high $\beta_{y}$-values a stronger effective kick can be achieved, hence structures positioned close to the vertically focusing quadrupoles are chosen.

During the simulations described below all bumps consist of the last structure before each of two consecutive vertically focusing quadrupoles. Each bump is controlled by two knobs. Turning one of these knobs gives an identical vertical offset to both of the structures, while turning the other one offsets them by the same amount but in opposite directions. In this way the knobs become almost independent, acting on the sine-phase and cosine-phase separately.

\section{SIMPLIFIED OPTIMISATION}

In order to get a first idea of how well the luminosity tuning bumps may be expected to perform, a first simulation was carried out using pure function optimisation.

The first step of this method is to calculate the initial particle coordinates at the interaction point for both the electron and the positron beam. The response of the particles to an adjustment of each of the knobs is then computed. All the tracking was performed using PLACET [3]. The data obtained can be used to tune one knob after the other with a standard linear minimisation method as in [4]. The first two knobs on the electron side were tuned first, then the first two on the other side. Then back again to the first side to tune the second bump and so on until all knobs had been moved to their optimal position. This routine was then repeated until 600 optimisation steps had been completed.

The luminosity was calculated as the convolution of two upright gaussians. Since the beams might be offset and might approach the IP with some angle the particle coordinates were corrected to have zero offset and angle before applying the equation. No beam-beam interaction was taken into account and the $\beta_{y}$ was assumed to be high, thereby neglecting the effect that the hourglass shape of the beam at the IP might have.

This simulation was performed for 50 different "machines" to obtain some statistics. A machine, in this context, denotes a line where the elements have firstly been scattered around a straight line, then the BPMs and the quadrupoles have been aligned using the ballistic align- 
ment method and finally the accelerating structures have been aligned using BPMs incorporated into them. The initial scattering has a gaussian distribution with:

- $\sigma=50 \mu m$ for quadrupoles

- $\sigma=10 \mu m$ for accelerating structures

- $\sigma=10 \mu m$ for the systematic error for the realignment of the accelerating structures

- $\sigma=10 \mu \mathrm{rad}$ for the vertical angle of the accelerating structures

- $\sigma=10 \mu m$ for BPMs

The effect of using different numbers of bumps was also investigated by repeating the simulations described above for 2, 3, 5 and 10 bumps respectively. The bumps were chosen such that the number of cavities separating them was more or less constant. All results can be found in Fig. 1.

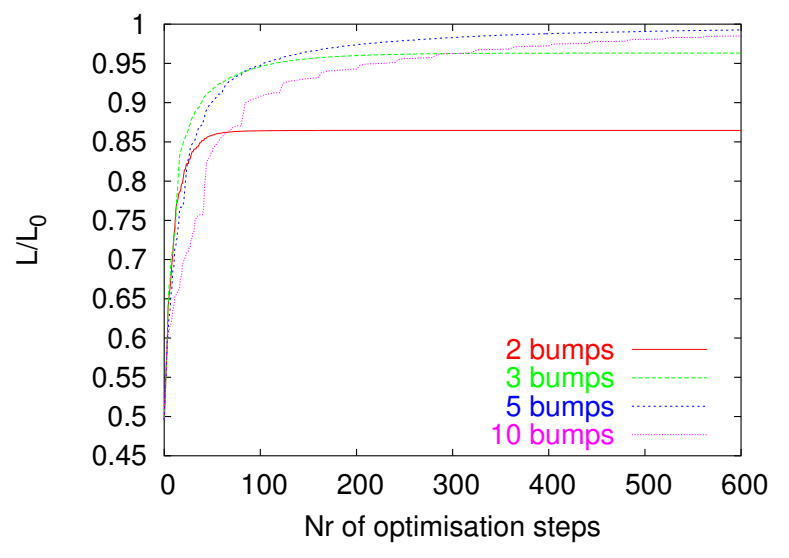

Figure 1: Average luminosity after each optimisation step. $2,3,5$ and 10 bumps were used. $L_{0}$ is the luminosity obtained by colliding two perfect beams.

\section{REALISTIC OPTIMISATION ROUTINE}

The optimisation routine described above is not very realistic, mainly because it does not take noise into account. To make a more realistic test of the luminosity tuning bumps the routine was slightly modified and noise was added to the luminosity measurement. The method is rather simple; each optimisation step is performed by trying five different knob settings and measuring the corresponding luminosity at the IP. Furthermore, random noise is added to the luminosity. This noise has a gaussian distribution with $\sigma=3 \%$. The noise distribution is also truncated at $3 \sigma$. By fitting a second-order polynomial to the data points obtained, a good approximation of the optimum knob setting is achieved. In order to avoid unsuccessful optimisations a few safety measures have been taken; in case the polynomial fit yields a positive second-order coefficient, in which case the extremum is a minimum, or if the optimal knob setting is too far from the current one, a couple of extra knob settings are tested. Further studies are required to see how this routine can be improved.

\section{SIMULATION OF LUMINOSITY TUNING BUMPS}

Identical simulations were carried out using the optimisation routine described above: PLACET was used for the tracking; the same 50 machines as before were used to get a statistical basis; the simulations were performed for $2,3,5$ and 10 bumps and 600 optimisation steps were used. First the new optimisation routine was tested without adding any noise, see Fig. 2. Then the routine was tested with the gaussian noise described above, see the results in Fig. 3. Note that here it is the actual luminosity that has been plotted. The noisy signal is used to obtain the optimum knob settings.

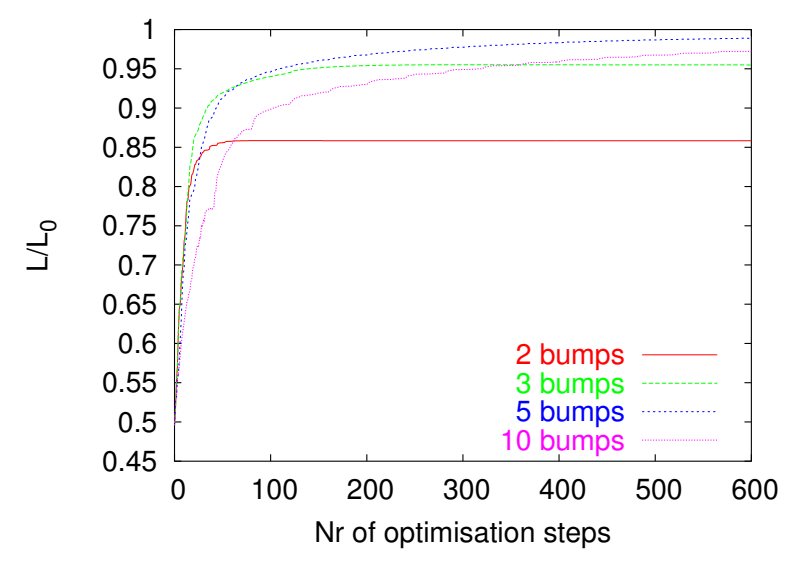

Figure 2: Luminosity after each optimisation step. $L_{0}$ is the luminosity obtained by colliding two perfect beams

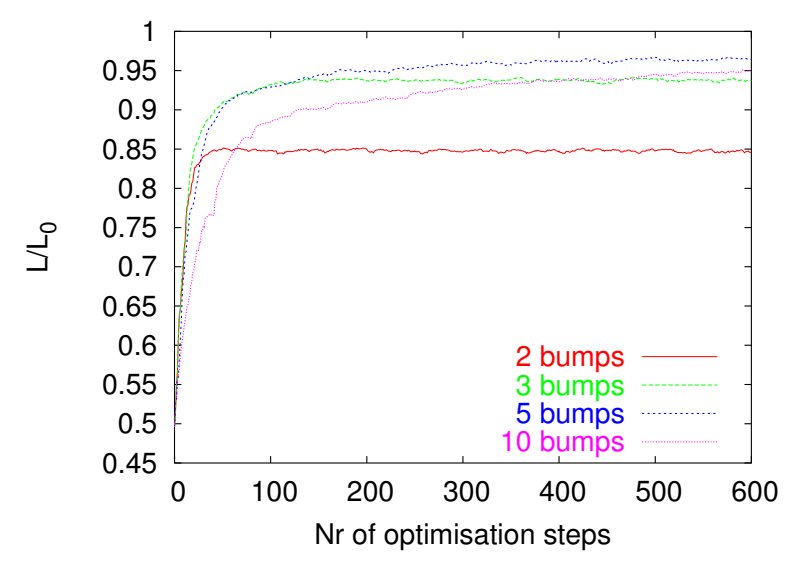

Figure 3: Luminosity after each optimisation step. The optimisation routine uses a luminosity signal with noise. $L_{0}$ is the luminosity obtained by colliding two perfect beams

\section{LASERWIRE SCANNER OPTIMISATION}

In previous studies the emittance had been minimised using emittance tuning bumps. It was assumed that the emittance was measured by determining the beam size at two 
locations separated by a betatron phase advance of $90^{\circ}$. The laserwires used for these measurements are conventionally smaller than the beam and the beam size is measured by scanning.

Here, the beam profile weighted with a gaussian distribution representing the target beam size is measured instead. This can be achieved using a laserwire that has a gaussian transverse profile of the proper size. One then tries to simply maximise the beam-laser luminosity.

The same optimisation routine as before was used to tune the knobs. Once again noise with a gaussian distribution was introduced in the luminosity signal. Since the beam might be offset compared to the laserwire after the initial scattering and alignment routines, the laserwire had to be moved to the position giving the highest photon count during the first step. This laserwire adjustment was also performed during the last optimisation step.

Despite the fact that only imperfections on one side of the IP have to be corrected in this case, Fig. 4 shows that the luminosities are comparable to or even lower than in Fig. 3. However, the plot also shows that at the end of the optimisation, when the laserwire position is adjusted, there is more luminosity to gain.

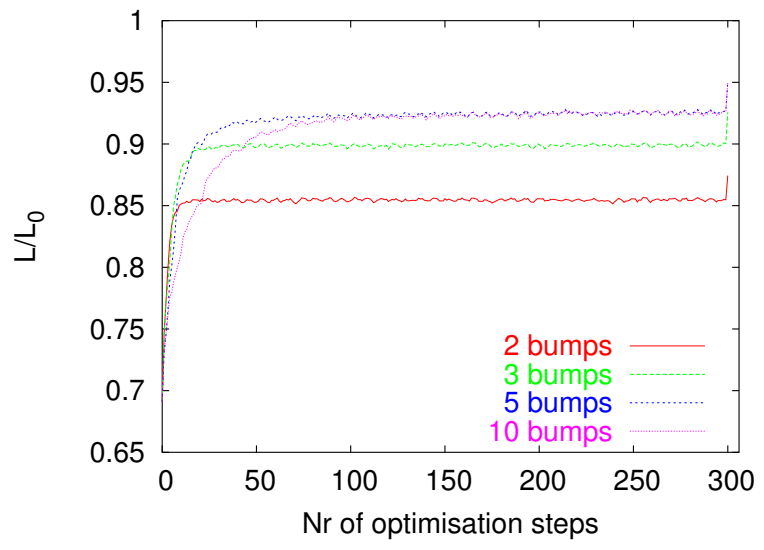

Figure 4: Luminosity measured with a laserwire with noise. The laserwire position was optimised at step 1 and 300 .

\section{TUNING OF LASERWIRE POSITION}

As can be seen in Fig. 4 the optimisation of the laserwire position had a strong effect on the measured luminosity. This indicates that a few more adjustments of the position should be done in order to make the optimisation more efficient. Two extra simulations were carried out in order to investigate this. During the first one the laserwire position was adjusted on every fifth step and during the second only on step 1, 5, 10, 20,40,80, 160 and 300. In both cases 5 bumps were used. Both of these simulations gave clearly better results than the previous one where the position was only adjusted during the first and last step, see Fig. 5. Obviously it is not necessary to optimise the position very often, but how often and when has to be further investigated.

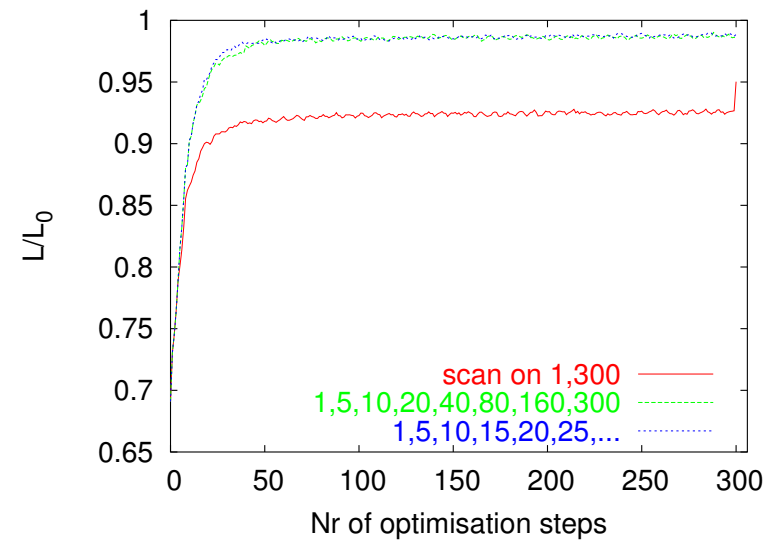

Figure 5: Luminosity measured with laserwire. The laserwire position is adjusted during the indicated optimisation steps. 5 bumps were used.

\section{CONCLUSION}

A method for optimisation of luminosity tuning bumps has been developed and tested. In an ideal situation, without noise in the luminosity measurements, almost all of the luminosity can be recovered using 5 bumps. More realistic tests show that almost $97 \%$ can be recovered when noise is added to the luminosity. The noise in this case had a gaussian distribution with $\sigma=3 \%$, truncated at $3 \sigma$. This test indicates that the luminosity tuning bumps are not very sensitive to noise.

Further simulations prove that a gaussian laserwire scanner can be used to optimise the luminosity tuning bumps on one side of the beam. In this case the laserwire is used to emulate a collision with a perfect beam. It turned out that adjustment of the laserwire position is important to optimise the bumps efficiently. It was shown that frequent adjustments are not necessary, but how often it has to be done should be further investigated.

In the near future extra luminosity losses due to the final focus system will be studied.

\section{ACKNOWLEDGEMENT}

This work is supported by the Commission of the European Communities under the $6^{\text {th }}$ Framework Programme "Structuring the European Research Area", contract number RIDS-011899.

\section{REFERENCES}

[1] D.Schulte, CLIC Note-370, 1998

[2] D.Schulte, CLIC Note-600, 2004

[3] http://dschulte.home.cern.ch/dschulte/placet.html

[4] William H. Press, et.al., "Numerical Recipes in Fortran", 2nd edition, 1992 